Commission. Prepared under Contract No. AT(11-1)68 for the San Francisco Operations Office, U. S。 Atomic Energy Commission.

†Work supported by the U.S. Atomic Energy Commission。

${ }^{1} J$. Weber, Phys. Rev. Lett. 22, 1320 (1969).

${ }^{2} \mathrm{~J}$. Weber, Phys. Rev. Lett. $\overline{24}, 276$ (1970).

${ }^{3}$ J. Weber, Phys. Rev. Lett. 25, 180 (1970).

${ }^{4}$ R. Davis, Jr., Phys. Rev。 Lett.12, 303 (1964).

${ }^{5}$ R. Davis, Jr., D. S. Harmer, and K. C. Hoffman, Phys。Rev。Lett.20, 1205 (1968)。

${ }^{6}$ W。 N. Charman, J. V。 Jelley, J。H. Fruin, G。A。 Baird, T. Delaney, B. G. Lawless, E. R. Hodgson, P.F.Scott, J. R. Shakeshaft, R. W。 P. Drever, W. P。 S. Meikle, R. A. Porter, and R. E. Spencer, Nature 228, 346 (1970).

'J. V. Jelley, at the Texas Symposium on Relativistic Astrophysics, Austin, Texas, December 1970 (to be published)。

${ }^{8} \mathrm{~B}$ 。 Partridge, private communication。

${ }^{9}$ J. N. Bahcall, Phys. Rev。136, B1164 (1964)。

${ }^{10} \mathrm{~J}$. N. Bahcall and S.C.Frautschi, Phys. Rev. 136, B1547 (1964).

${ }^{11}$ W. D. Arnett, High Energy Astrophysics, edited by C. De Witt, E. Schatzman, and P. Veron (Gordon and Breach, New York, 1967), Vol。3; see especially p. 142 .

${ }^{12} \mathrm{~J}$. N. Bahcall and R. H. Ulrich, Astrophys。J.160, 157 (1970).

${ }^{13}$ J. N. Bahcall, Phys。Rev。135, B137 (1964)。

${ }^{14} \mathrm{~J} . \mathrm{N}$. Bahcall, Phys. Rev。 Lett. 17, 398 (1966).

${ }^{15} \mathrm{G}$. W. Gibbons and S. W. Hawking, to be published.

${ }^{16} \mathrm{R}$. Ruffini and J. A. Wheeler, in "The Significance of Space Research for Fundamental Physics" (European Space Research Organization, Paris, France, to be published).

\title{
Interplanetary Deceleration of Solar Cosmic Rays*
}

\author{
S. S. Murray, $†$ E. C. Stone, and R. E. Vogt \\ California Institute of Technology, Pasadena, California 91109
}

(Received 25 January 1971)

\begin{abstract}
Observations of solar-flare proton fluxes of low energies $(1-10 \mathrm{MeV})$ during the 7 June 1969 event have been used to study the effects of energy-change processes on particles propagating in interplanetary space. It is found that the proton energies are decreasing with time at a rate which is consistent with an exponential-decay time constant of $210 \pm 10 \mathrm{~h}$. Since adiabatic deceleration in a uniform solar wind would result in a faster decay $(78 \pm 4 \mathrm{~h}$ ), additional processes such as Fermi acceleration or a more general deceleration process must be considered.
\end{abstract}

The propagation of charged particles through the interplanetary medium may be described by a Fokker-Planck transport equation including terms for diffusion, convection, and energy change in the expanding solar wind. The general formulation of particle propagation, as introduced by Parker $^{1}$ in 1965 , included a term accounting for adiabatic deceleration in a uniformly expanding solar wind. Parker also considered for relativistic particles the effects of Fermi acceleration in the solar wind and concluded that this process was unimportant.

There has been only limited success in obtaining analytical solutions to the cosmic-ray transport equation with convection and adiabatic deceleration processes included. Fisk and Axford ${ }^{2}$ obtained a solution for solar-flare propagation based on special forms for the diffusion coefficient and boundary conditions which are not generally applicable. More recently Forman ${ }^{3}$ has obtained a solution based on the assumption that diffusive effects and density gradients are small and that convection and adiabatic deceler- ation processes dominate the decay phase of solar-flare proton events. However, there had been to date no direct experimental evidence for the existence of an energy-change process occurring in interplanetary space. In this paper we present data which provide strong evidence for energy-change processes.

It has been suggested ${ }^{3}$ that the effects of an energy-change process can be observed experimentally during the decay phase of a solar-flare proton event provided that there is a distinct feature in the proton differential density spectrum. A variation with time of the characteristic energy associated with the feature would be evidence for an energy-change process.

It should be noted that in the absence of a distinct feature the effects of energy change could not be as easily separated from the effects of diffusion and convection. Similarly, if the feature is indistinct as a result of inadequate statistical accuracy or energy resolution, then an energy change of only 10 to $50 \%$ per day would not be detected. However, the measurements reported 


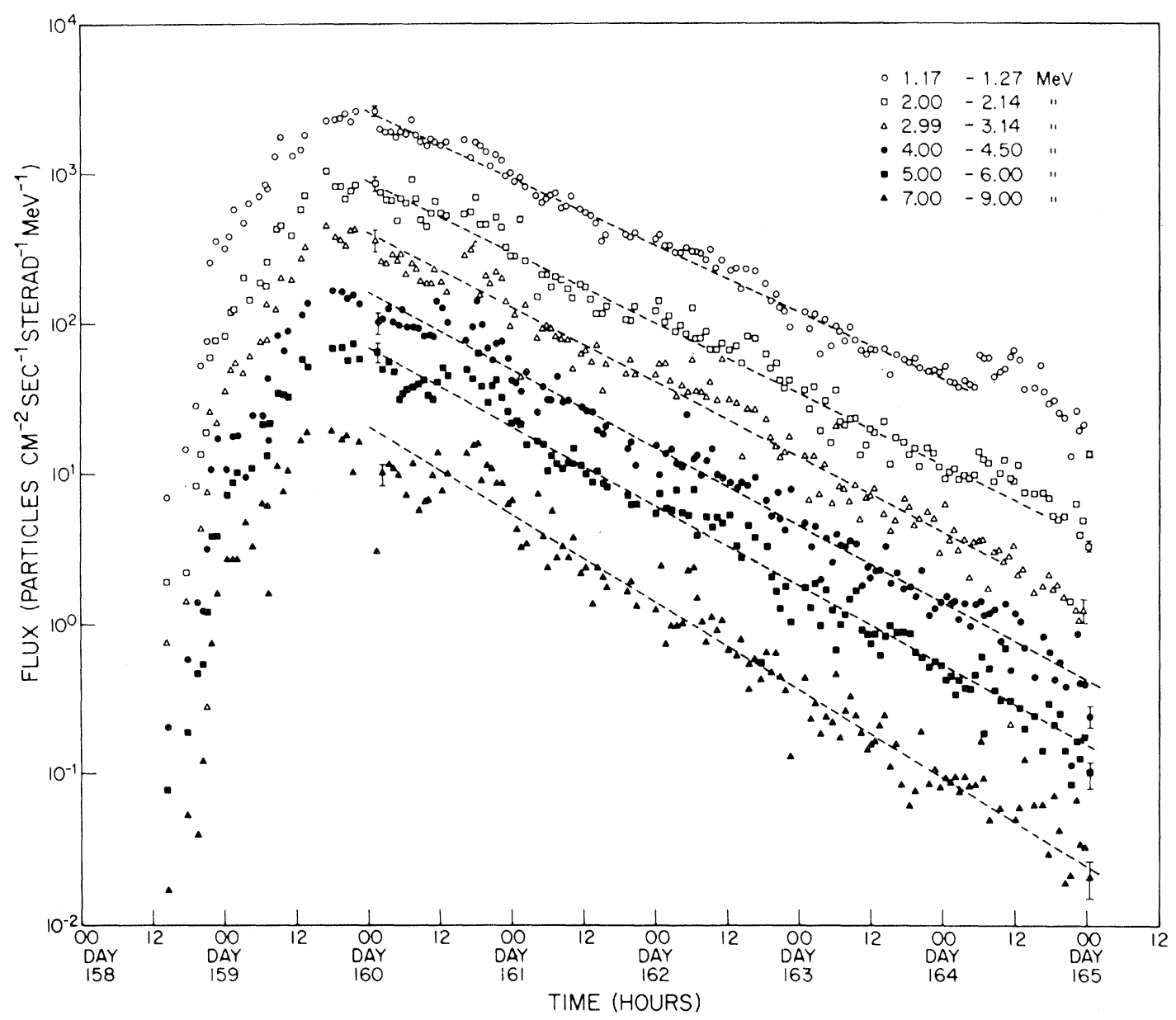

FIG. 1. Fluxes of protons in various energy intervals versus universal time for the 7 June 1969 (day 158) solarflare event. The dashed lines are exponential fits to the decays of the fluxes.

here are precise enough to permit us to study the time dependence of a distinct spectral feature which was observed during the decay phase of the 7 June 1969 solar-flare event.

The observations of the fluxes of $1-$ to $10-\mathrm{MeV}$ solar-flare protons reported here were made with the California Institute of Technology solar and galactic cosmic-ray experiment on OGO-6, a low altitude polar-orbiting satellite. The experiment, which has been described in detail elsewhere, ${ }^{4}$ includes a charged-particle telescope in which particle type and energy are determined by a simultaneous measurement of energy loss and total energy in fully depleted, gold-silicon surface barrier solid-state detectors. There is unambiguous separation of protons and $\alpha$ particles above $\sim 3.3-\mathrm{MeV}$ total energy, and the extrapolated correction for $\alpha$-particle contamination of the proton fluxes below $\sim 3.3 \mathrm{MeV}$ is negligible during this event.

This proton event was probably associated with a solar flare on the east limb of the solar disk in the McMath plage region 10135 at $45^{\circ} \mathrm{E}$ longitude and $15^{\circ} \mathrm{S}$ latitude. ${ }^{5}$

In Fig. 1, the differential flux of protons versus universal time is plotted for several of the energy intervals included in this analysis. During the smooth decay phase of this event, we observe essentially exponential decreases in the flux as a function of time. We have chosen the 3day period from 0227 UT on day 161 through 0256 UT on day 164 as the interval over which to analyze the data. For this phase of the event, we have fitted exponential decay functions to the observed differential fluxes. We found that the decay time constant decreased with increasing energy from $\sim 23 \mathrm{~h}$ at $\sim 1 \mathrm{MeV}$ to $\sim 18 \mathrm{~h}$ at $\sim 10 \mathrm{MeV}$.

In Fig. 2, several examples of the proton differential density spectra are shown for high-latitude (polar) data samples taken at roughly 24-h intervals during the 3 days of interest. Good fits to these spectra have been obtained by fitting 


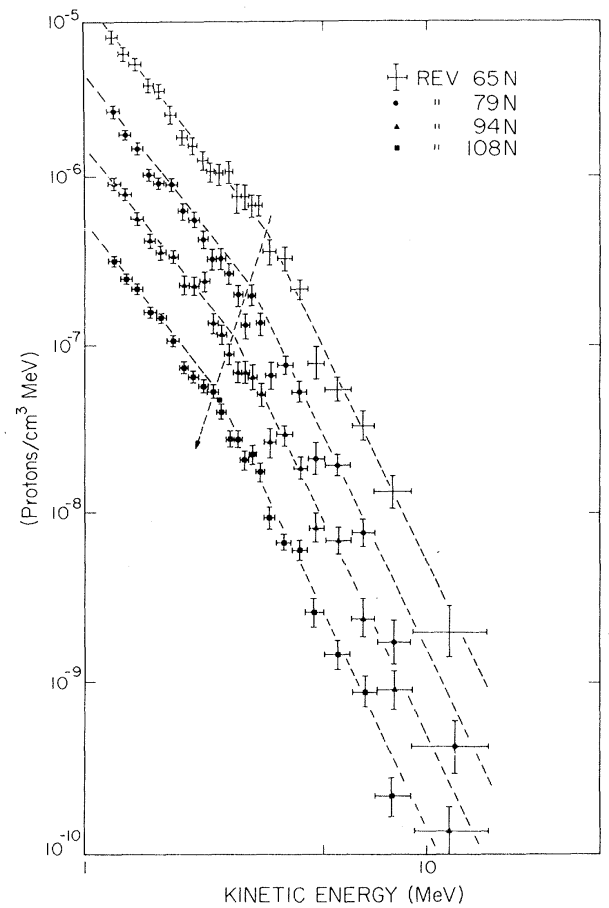

FIG. 2. Differential density spectra of protons during the decay phase of the 7 June 1969 event. The data for each spectrum were accumulated at $\sim 24-\mathrm{h}$ intervals during the north-polar pass of the indicated satellite revolution. The arrow indicates the time dependence of the intersection of the two power laws which are fit to each spectrum.

each spectrum with two power-law functions of kinetic energy $T$ and universal time $t$ :

$$
\begin{array}{ll}
U_{a}(T, t)=N_{a}(t) T^{-2.6}, & T<2.5 \mathrm{MeV} ; \\
U_{b}(T, t)=N_{b}(t) T^{-4.2}, & T>4.0 \mathrm{MeV} .
\end{array}
$$

The energy $T_{\text {int }}$ at which these power-law functions intersect is a characteristic feature of the differential density spectrum which can be determined as a function of $t$.

We have fitted the differential density spectra observed for each of the 72 polar passes of the satellite during the above three-day interval by the two power-law functions given by Eq. (1), varying only $N_{a}$ and $N_{b}$. For all of these spectra, the goodness-of-fit parameter $\chi^{2}$ is typically between 0.5 and 1.0, which indicates that these power-law functions are indeed valid representations of the spectrum over the interval of interest.

In Fig. 3, the intersection energy $T_{\text {int }}$ of these functions is plotted against universal time $t$. The solid line shown is the best fit of the data by an exponential function of the form

$$
T_{\text {int }}(t)=T_{\text {int }}\left(t_{0}\right) \exp \left(-t / \tau_{E}\right) \text {. }
$$

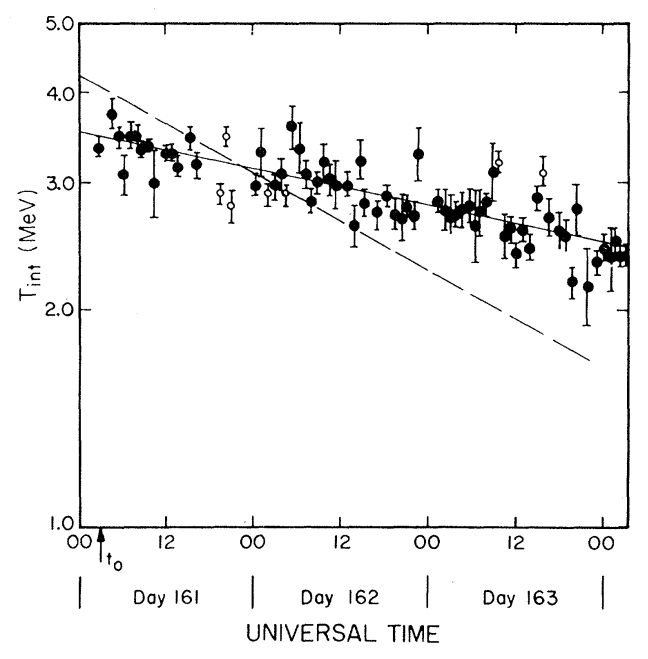

FIG. 3. Time dependence of the intersection energy of the two power laws which are fitted to each of the 72 differential density spectra observed during the period at $t_{0}=0227 \mathrm{UT}$ on day 161 . The solid line is an exponential fit to these data, excluding the open circles. The dashed line is the expected energy change due to adiabatic deceleration as given by Eqs. (6) and (7).

The values of the parameters obtained from the fit are

$$
\tau_{E}=210 \pm 10 \mathrm{~h}, \quad T_{\text {int }}\left(t_{0}\right)=3.5 \pm 0.01 \mathrm{MeV} .
$$

We believe that these are the first experimental results which directly indicate the occurance of an energy-change process during charged-particle propagation in interplanetary space. Of course, it may be possible to explain these results by invoking either a special source function or a special time-varying diffusion process.

However, neither of these currently has a strong physical or theoretical basis. We will, therefore, proceed to discuss these results within the context of current propagation models involving energy-change processes.

If the time dependence of the intersection energy $T_{\text {int }}$ is the exponential dependence contained in Eq. (2), then the energy-change process is of the form

$$
d T / d t=-T / \tau_{E},
$$

and the complete transport equation for these particles is given by

$$
\frac{\partial U}{\partial t}=\nabla \cdot(\vec{\kappa} \nabla U)-\nabla \cdot(U \overrightarrow{\mathrm{V}})+\frac{1}{\tau_{E}} \frac{\partial(U T)}{\partial T},
$$

where $\vec{K}$ is the diffusion tensor and $\vec{V}$ the velocity of the solar wind.

The transport equation proposed by Parker ${ }^{1}$ is of the same form, with the energy-change term 
due to adiabatic deceleration in a uniform solar wind given by

$$
d T / d t=-T / \tau_{\mathrm{ad}},
$$

where $\tau_{\mathrm{ad}}=3 r / 4 \mathrm{~V}$ and $r$ is the distance from the sun to the point of observation, i.e., $1 \mathrm{~A}$.U. for the present observation.

Evaluating $\tau_{\text {ad }}$ during this event, using $V=400$ $\pm 20 \mathrm{~km} / \mathrm{sec}$ as measured by the Vela satellites, ${ }^{5}$ we find that

$$
\tau_{\text {ad }}=78 \pm 4 \mathbf{h}_{2}
$$

which is significantly smaller than the observed time constant

$$
\tau_{E}=210 \pm 10 \mathrm{~h} .
$$

We interpret this discrepancy as an indication that either the effects of adiabatic deceleration are not adequately described by Eq. (6), or that there is a competing acceleration process. If such an acceleration process could be described by an exponential time constant $\tau_{A}$, then the observed time constant would be given by

$$
\tau_{E}{ }^{-1}=\tau_{\mathrm{ad}}{ }^{-1}-\tau_{A}{ }^{-1} \text {. }
$$

For this event we find that

$$
\tau_{A}=125 \pm 10 \mathrm{~h} \text {. }
$$

Jokipii ${ }^{6}$ has recently shown that Fermi acceleration in the solar wind is an attractive possibility for such an acceleration mechanism. However, more observations must be analyzed before a complete evaluation of interplanetary acceleration and deceleration processes is possible.

We are grateful to J. R. Jokipii for discussing his results with us prior to publication. We also appreciate valuable discussions with L. Davis, Jr., J. L. Fanselow, and D. S. Intriligator.

*Work supported by the National Aeronautics and Space Administration under Contract No. NAS5-9312 and Grants No. NGR-05-002-160 and No. NGL-05-002007.

$\uparrow$ Present address: American Science \& Engineering, Cambridge, Mass. 02142.

${ }^{1}$ E. N. Parker, Planet. Space Sci. 13, 9 (1965).

${ }^{2}$ L. A. Fisk and W. I. Axford, J. Geophys. Res. $\underline{73}$, 4396 (1968).

${ }^{3}$ M. A. Forman, J. Geophys. Res. 75, 3147 (1970).

${ }^{4}$ W. E. Althouse, E. C. Stone, R. E. Vogt, and T. H. Harrington, IEEE Trans. Nucl. Sci. 15, 229 (1967).

${ }^{5}$ Sol.-Geophys. Data No. 299, Part I (1969), and No. 300, Part I (1969), and No. 304, Part II (1969), and No. 305, Part II (1970).

${ }^{6} \mathrm{~J}$. R. Jokipii, following Letter [Phys. Rev. Lett. 26 , $666(1971)]$.

\title{
Deceleration and Acceleration of Cosmic Rays in the Solar Wind*
}

\author{
J. R. Jokipii † \\ Physics Department, California Institute of Technology, Pasadena, California 91109 \\ (Received 25 January 1971)
}

\begin{abstract}
Recent observations of the deceleration of low-energy solar cosmic rays in the solar wind are discussed in the context of cosmic-ray transport theory. It is concluded that the rate of deceleration is much slower than would be produced by adiabatic energy: change due to the observed expansion of the plasma, so that a competing acceleration process must be operative. Second-order Fermi acceleration by hydromagnetic waves, acting together with adiabatic energy change, is shown to provide a natural interpretation of the observations.
\end{abstract}

Recent observations ${ }^{1}$ have clearly isolated an energy-loss process occurring in the transport of solar cosmic rays in the solar wind. The rate of energy change of $\sim 3-\mathrm{MeV}$ protons at Earth, obtained by following a characteristic feature of the energy spectrum as a function of time, is given by $^{1}$

$$
d T / d t=-T /(210 \pm 10 \mathrm{~h}),
$$

where $T$ is the kinetic energy. Interpretation of the result in terms of processes not involving energy change appears to be quite unlikely. ${ }^{1}$
It is the purpose of this Letter to consider the interpretation of the above result in the context of cosmic-ray transport theory. The observation is found to fit very well into the standard theory, but only if one admits that Fermi acceleration is important for protons with energies of the order of 1-10 MeV.

Theoretical studies of cosmic-ray transport in the solar wind generally proceed from the Fokker-Planck equations ${ }^{2,3}$ first derived by Parker. If $U(\vec{r}, t, T)$ is the cosmic-ray density as a function of position, time, and kinetic energy; if $\kappa_{i j}$ 